\section{Estrés y desempeño académico en estudiantes de Odontología}

\author{
Stress and academic achievement in students of dentistry
}

\author{
Teresa Evaristo-Chiyong', \\ Sylvia Chein-Villacampa ${ }^{1}$
}

\begin{abstract}
Resumen
El propósito de este estudio fue evaluar el estrés académico y su repercusión con el desempeńo académico de los estudiantes de odontología. Metodología: se trabajó con una muestra probabilística de 186 estudiantes de odontología que cursaron el semestre académico 2014-1, distribuidos de forma estratificada por ańo de estudios: primero $(n=37)$; $\operatorname{segundo}(n=29)$; tercero $(n=44)$; $\operatorname{cuarto}(n=36)$ y quinto $(n=40)$ y seleccionados de manera aleatoria simple. La medición de la variable estrés académico se realizó a través del cuestionario Inventario SISCO validado, que evalúa: estresores académicos, manifestaciones del estrés y las estrategias de afrontamiento y que obtuvo una consistencia interna de 0.768 . La variable desempeño académico se midió a través de la nota promedio del estudiante al término del semestre 2014-1. Para el análisis estadístico se utilizó pruebas estadísticas bivariadas y multivariadas. Resultados y conclusiones: se encontró que el 100\% de los estudiantes presentaron en algún momento situación de estrés con una intensidad promedio de 3.5. El rendimiento académico promedio fue de 13.21(basado en el sistema vigesimal 0-20). Los estudiantes de años de estudio superiores presentaron un mayor rendimiento académico, no evidenciándose diferencias estadísticamente significativas según sexo. En el análisis multivariado se halló que la habilidad asertiva es el factor determinante en el rendimiento académico de los estudiantes de odontología (Beta $0.167, \mathrm{p}<0.05$ ).
\end{abstract}

Palabras clave: Estrés académico; desempeño académico; estudiantes; odontología.

\section{Abstract}

The purpose of this study was to evaluate academic stress and its impact in the academic performance of students of dentistry. Methodology: we worked with a probabilistic sample of 186 dental students who completed the semester 2014-1, stratified by year of study: first $(n=37)$; second $(n=29)$; third $(\mathrm{n}=44)$; fourth $(\mathrm{n}=36)$ and fifth $(\mathrm{n}=40)$ and single randomly selected. The measurement of academic stress variable was performed through the SISCO inventory validated questionnaire, which assesses: academic stressors, manifestations of stress and coping strategies and obtained an internal consistency of 0.768 . The academic performance variable was measured by the average student at the end of the semester 2014-1. For statistical analysis, bivariate and multivariate statistical tests were used. Results and conclusions: it was found that $100 \%$ of the students presented sometime stressful situation with an average intensity of 3.5. The average academic performance was 13.21. Students in the senior years of study have a higher academic performance, demonstrating no statistically significant differences by sex. It was found in the multivariate analysis that assertive ability is the determining factor in academic performance of students of dentistry (Beta 0.167, p <0.05).

Keywords: Academic stress; academic performance; students; dentistry.

\section{Artículo Original}

1. Departamento Académico de Estomatología Preventiva y Social de la Facultad de Odontología de la Universidad Nacional Mayor de San Marcos, Perú.

\section{Correspondencia}

Mg. Teresa Evaristo-Chiyong

Facultad de Odontología. Universidad Nacional Mayor de San Marcos. Av. Germán Amézaga 375, Lima 1, Perú.

Correo electrónico: t_evaristo2002@yahoo.es

Coautora:

Chein: scheinv@unmsm.edu.pe

\section{Introducción}

El desempeño del estudiante dentro del proceso de formación universitaria constituye un factor imprescindible en el abordaje del tema de la calidad en la educación superior, debido a que es un indicador que permite una aproximación a la realidad educativa.

Este desempeño medido a través del rendimiento académico al ser multicausal, envuelve una enorme capacidad explicativa de los distintos factores y espacios temporales que intervienen en el proceso de aprendizaje. Diferentes aspectos se asocian al rendimiento académico, entre los que destacan el componente personal, social e institucional.

Los estresores académicos son todos aquellos factores o estímulos del ambiente organizacional educativo que presionan o sobrecargan al estudiante entre los que se pueden destacar las evaluaciones, las relaciones sociales, la sobrecarga de trabajo, metodología docente, entre otros. Un elevado nivel de estrés originado por los distintos estresores académicos altera el triple sistema de respuestas, cognitivo-motorfisiológico, lo que podría tener una re- percusión negativa en el rendimiento académico.

Este trabajo servirá como sustento teórico que permita plantear estrategias apropiadas en el proceso educativo del estudiante, que garantice una mejor formación profesional y desarrollo personal social.

Berrío GN, ${ }^{1}$ Díaz $\mathrm{CS}^{2}$ y Casuso $\mathrm{HJ}^{3}$ plantean que el estrés académico es un fenómeno cognitivo, fisiológico y comportamental de elevada frecuencia en los estudiantes y en el ámbito académico representa un determinante fundamental que reduce el desempeño 
y cuyos factores desencadenantes pueden provenir de múltiples fuentes .

Los niveles de estrés y sus manifestaciones en estudiantes de odontología es alta, superando el 90\% según Díaz CS², Maruris $\mathrm{RM}^{4}$ y Bedoya $\mathrm{SA}^{5}$, siendo las principales situaciones generadoras de estrés el tiempo para cumplir con las actividades, sobrecarga de tareas, trabajos de cursos y responsabilidad por cumplir obligaciones académicas.

El objetivo del estudio fue evaluar el estrés académico y su relación con el desempeño académico de los estudiantes de la Facultad de Odontología de la Universidad Nacional Mayor de San Marcos (UNMSM).

\section{Materiales y Método}

El diseño corresponde a un estudio observacional, descriptivo, transversal. La muestra estuvo conformada por 186 estudiantes de la facultad de odontología de la UNMSM matriculados en el semestre 2014-I y obtenidos a través de un muestreo probabilístico estratificado distribuidos por año de estudios: Primero(n=37); Segundo: $(n=29)$ Tercero $(n=44)$; Cuarto $(n=36)$ y Quinto $(n=40)$. La selección de cada estudiante se realizó de manera aleatoria simple, excluyéndose a aquellos estudiantes que cursaban 3 o menos asignaturas.

La medición de la variable estrés académico se realizó a través del cuestionario Inventario SISCO validado y que obtuvo una consistencia interna a través de la prueba alfa de Conbrach de 0.768 . Este instrumento evalúa tres dimensiones del estrés: estresores académicos (8 preguntas) manifestaciones del estrés (15 preguntas) y las estrategias de afrontamiento (6 preguntas). Cada una de las preguntas puntuadas del 1 al 5, en una escala ordinal tipo Likert. La variable desempeño académico se midió a través del registro de la nota promedio del estudiante al término del semestre 2014-1 proporcionada por la Escuela académica profesional.

El análisis de datos se realizó haciendo uso del paquete estadístico SPSS versión 21. El análisis descriptivo de los mismos se realizó a través de tablas de frecuencia y medidas de tendencia central y dispersión de acuerdo a la naturaleza de las variables de estudio.

Para el análisis inferencial se utilizaron pruebas bivariadas tales como, correlación de Spearman, Kruskall Wallis y Anova; asimismo se realizó el análisis multivariado de regresión lineal.

\section{Resultados}

De los 186 estudiantes encuestados el $100 \%$ refirió presentar estrés con una intensidad de 3.5 , sobre una escala de 1 a 5. Los estresores que se presentaron con mayor frecuencia entre las categorías "casi siempre" y "siempre" son: sobrecarga de tareas y trabajos, seguido por el tiempo limitado y las evaluaciones. Un 57\% considera que "algunas veces” la personalidad y carácter del profesor es considerado un factor estresor, seguido por el factor participación en clase en un $47.8 \%$. (Tabla 1 y 2 ). Las principales reacciones ante el estrés que se presentaron entre las categorías "casi siempre" y "siempre" son: somnolencia y mayor necesidad de dormir en un $50 \%$, seguido por fatiga crónica en un $36.6 \%$. Al relacionar según ańo de estudio, se observó que los trastornos del sueño, inquietud, conflicto o tendencia a polemizar y aumento del consumo de alimentos difieren según año de estudio. (Tabla 3 y 4 )

Las estrategias de afrontamiento que presentan los estudiantes ante el estrés entre las categorías "casi siempre" y "siempre" son: habilidad asertiva $51.6 \%$, seguido de elaboración de un plan $38.7 \%$. No encontrándose diferencias estadísticamente significativas entre estas según año de estudio. (Gráfico 1). El rendimiento académico promedio fue de $13.21 \pm 1.04$, en base vigesimal $0-20$. Los estudiantes de los mayores ańos de estudio presentan un mayor rendimiento académico, estadisticamente significativo según sexo, y según año. La intensidad del estrés fue de 3.5 \pm 0.93 . No encontrándose diferencias estadísticamente significativas en relación al año de estudio (Tabla 5). La intensidad del estrés de los estudiantes no se encuentra relacionada con el rendimiento académico de los mismos. (Tabla 6). Al realizar el análisis de regresión se evidenció que la habilidad asertiva es el único factor dentro de las dimensiones de estrés asociado al rendimiento académico de los estudiantes (Tabla 7).

\section{Discusión}

En el presente estudio la presencia de estrés fue del $100 \%$, dato muy próximo al encontrado por Bedoya $a^{5}$, en estudiantes de odontología donde la prevalencia de estrés fue del 96.6\%. Autores como Díaz ${ }^{2}$, Vallejo ${ }^{6}$ y Rafael $^{7}$ concluyen que la presencia de estrés es un fenómeno generalizado entre los estudiantes universitarios.

$\mathrm{Al}$ analizar las situaciones generadoras de estrés, se evidenció que las que se presentan con mayor frecuencia en las categorías "siempre" y "casi siempre" fueron la sobrecarga de tareas, el tiempo limitado y las evaluaciones. Estos resultados son coincidentes con Díaz ${ }^{2}$, Bedoya $^{5}$ y Del Toro ${ }^{8}$, quienes evidenciaron que la sobrecarga de tareas y el tiempo limitado fueron las principales generadoras de estrés. Es importante destacar que las evaluaciones no son la principal fuente de estrés para el estudiante sino la relación trabajo por realizar y tiempo para desarrollarlo. Considerando que en promedio el plan de estudio de los estudiantes de odontología en cada semestre está compuesto por seis asignaturas obligatorias independientes una de otras con respecto a la evaluación y desarrollo de trabajos, y que el horario para el desarrollo de las mismas genera que el estudiante permanezca en promedio 12 horas dentro de los ambientes universitarios de lunes a sábado, no es de sorprender estos resultados, que evidencian la posible sobrecarga de trabajos y el tiempo que tienen para desarrollarlos.

Con respecto a la personalidad del profesor como factor estresante, Alves y Acevedo ${ }^{9}$ plantean que el rendimiento académico es "el resultado del proceso de aprendizaje, a través del cual el docente en conjunto con el estudiante pueden determinar en qué cantidad y calidad, el aprendizaje facilitado, ha sido interiorizado por este último. El profesor, orientador, tutor del estudiante, en consecuencia debe cumplir un rol mediador en el aprendizaje del estudiante, y éste rol difícilmente se cumplirá si la personalidad del mismo genera más una situación de estrés que de confianza y seguridad para el estudiante.

Las principales reacciones ante el estrés presentadas por los estudiantes en las categorías "siempre" y "casi siempre" fueron somnolencia y mayor necesidad de dormir, seguido por fatiga crónica, estos resultados son coincidentes con Diaz ${ }^{2}$ quién encontró que los trastornos del sueño fueron los principales reacciones. La presencia de ansiedad, angustia y desesperación presentan frecuencia elevada entre las categorías "algunas veces" y "casi siempre", manifestaciones psicológicas que como plantean Díaz ${ }^{2}$, Bedoya ${ }^{5}$ y Vallejo ${ }^{6}$ se encuentran presentes en estudiantes universitarios. 
Tabla 1. Estresores académicos en los estudiantes de odontología de la UNMSM-2014

\begin{tabular}{llllll}
\hline Estresores & Nunca & Rara vez & Algunas veces & Casi siempre & Siempre \\
\hline Competencia con compañeros $\left(^{*}\right)$ & $10(5.4 \%)$ & $52(28 \%)$ & $85(45.7 \%)$ & $34(18.3 \%)$ & $5(2.7 \%)$ \\
Sobre carga de tareas y trabajos & $0(0 \%)$ & $9(4.8 \%)$ & $49(26.3 \%)$ & $91(48.9 \%)$ & $37(19.9 \%)$ \\
Personalidad y carácter del profesor & $5(2.7 \%)$ & $48(25.8 \%)$ & $106(57 \%)$ & $22(11.8 \%)$ & $5(2.7 \%)$ \\
Evaluaciones & $0(0 \%)$ & $17(9.2 \%)$ & $59(31.9 \%)$ & $76(41.1 \%)$ & $33(17.8 \%)$ \\
Tipo de trabajos & $2(1.1 \%)$ & $14(7.5 \%)$ & $84(45.2 \%)$ & $57(30.6 \%)$ & $29(15.6 \%)$ \\
No entender temas abordados & $12(6.5 \%)$ & $66(35.5 \%)$ & $72(38.7 \%)$ & $28(15.1 \%)$ & $8(4.3 \%)$ \\
Participación en clase & $14(7.5 \%)$ & $51(27.4 \%)$ & $89(47.8 \%)$ & $27(14.5 \%)$ & $5(2.7 \%)$ \\
Tiempo limitado & $5(2.7 \%)$ & $16(8.6 \%)$ & $53(28.5 \%)$ & $81(43.5 \%)$ & $31(16.7 \%)$ \\
\hline
\end{tabular}

Tabla 2. Estresores académicos según año de estudios en los estudiantes de odontología de la UNMSM-2014

\begin{tabular}{|c|c|c|c|c|c|c|}
\hline \multirow[b]{2}{*}{ Estresores } & & \multicolumn{5}{|c|}{ Año de estudios } \\
\hline & & Primero & Segundo & Tercero & Cuarto & Quinto \\
\hline \multirow[t]{2}{*}{ Competencia con compañeros } & $\bar{X}$ & 2,89 & 2,86 & 3,25 & 2,44 & 2,73 \\
\hline & $\sigma$ & ,936 & 875 & ,686 & ,773 & ,933 \\
\hline \multirow[t]{2}{*}{ Sobre carga de tareas y trabajos } & $\bar{x}$ & 3,95 & 3,76 & 3,84 & 3,92 & 3,73 \\
\hline & $\sigma$ & ,815 & ,786 & ,861 & ,770 & ,751 \\
\hline \multirow[t]{2}{*}{ Personalidad y carácter del profesor } & $\overline{\mathrm{X}}$ & 2,81 & 2,90 & 3,02 & 2,75 & 2,80 \\
\hline & $\hat{\sigma}$ &, 739 & 900 &, 664 & 874 & 648 \\
\hline \multirow[t]{2}{*}{ Evaluaciones $\left({ }^{*}\right)$} & $\bar{x}$ & 3,86 & 3,69 & 3,81 & 3,53 & 3,48 \\
\hline & $\sigma$ & ,855 & ,891 & ,824 & ,941 & 847 \\
\hline \multirow[t]{2}{*}{ Tipo de trabajos } & $\bar{x}$ & 3,59 & 3,21 & 3,82 & 3,25 & 3,60 \\
\hline & $\sigma$ & ,956 & 861 & ,815 & ,937 & ,744 \\
\hline \multirow[t]{2}{*}{ No entender temas abordados $\left({ }^{* *}\right)$} & $\bar{x}$ & 3,16 & 2,72 & 2,82 & 2,44 & 2,60 \\
\hline & $\sigma$ & 1,014 & ,882 & ,922 & ,909 & 841 \\
\hline \multirow[t]{2}{*}{ Participación en clase } & $\bar{x}$ & 2,62 & 2,83 & 3,09 & 2,67 & 2,63 \\
\hline & $\sigma$ & ,893 & ,889 & ,884 & ,793 & 897 \\
\hline \multirow[t]{2}{*}{ Tiempo limitado } & $\overline{\mathrm{X}}$ & 3,57 & 3,45 & 3,73 & 3,58 & 3,75 \\
\hline & $\sigma$ & ,899 & ,948 & ,817 & 1,131 & 981 \\
\hline
\end{tabular}

Tabla 3. Reacciones ante el estrés en los estudiantes de odontología de la UNMSM-2014

\begin{tabular}{|c|c|c|c|c|c|}
\hline & Nunca & Rara vez & Algunas veces & Casi siempre & Siempre \\
\hline Trastornos del sueño $\left(^{*}\right)$ & $35(18.8 \%)$ & $62(33.3 \%)$ & $52(28 \%)$ & $32(17.2 \%)$ & $5(2.7 \%)$ \\
\hline Fatiga crónica & $16(8.6 \%)$ & $22(11.8 \%)$ & $80(43 \%)$ & $52(28.0 \%)$ & $16(8.6 \%)$ \\
\hline Problemas digestivos,abdominales,diarrea & $46(24.7 \%)$ & $62(33.3 \%)$ & $57(30.6 \%)$ & $17(9.1 \%)$ & $4(2.2 \%)$ \\
\hline Rascarse,moders uñas,frotarse & $50(26.9 \%)$ & $46(24.7 \%($ & $51(27.4 \%)$ & $29(15.6 \%)$ & $10(5.4 \%)$ \\
\hline Somnolencia,mayor necesidad de dormir & $8(4.3 \%)$ & $26(14 \%)$ & $59(31.7 \%)$ & $59(31.7 \%)$ & $34(18.3 \%)$ \\
\hline Sentimientos de depresión y tristeza & $23(12.4 \%)$ & $63(33.9 \%)$ & $66(35.5 \%)$ & $27(14.5 \%)$ & $7(3.7 \%)$ \\
\hline Ansiedad, angustia desesperación & $31(16.7 \%)$ & $37(19.9 \%)$ & $73(39.2 \%)$ & $37(19.9 \%)$ & $8(4.3 \%)$ \\
\hline Problemas de concentración & $10(5.4 \%)$ & $49(26.3 \%)$ & $83(44.6 \%)$ & $36(19.4 \%)$ & $8(4.3 \%)$ \\
\hline Sentimientos de agresividad o aumento de irritabilidad & $31(16.7 \%)$ & $58(31.2 \%)$ & $69(37.1 \%)$ & $24(12.9 \%)$ & $4(2.2 \%)$ \\
\hline Conflicto o tendencia a polemizar o discutir $\left(^{* * *}\right)$ & $30(16.1 \%)$ & $66(35.5 \%)$ & $75(40.3 \%)$ & $13(7 \%)$ & $2(1.1 \%)$ \\
\hline Otros (frustación,deseo abandonar la carrera,varicela) & $0(0 \%)$ & $0(0 \%)$ & $4(100 \%)$ & $0(0 \%)$ & $0(0 \%)$ \\
\hline
\end{tabular}

Gráfico 1. Estrategias de afrontamiento al strés de los estudiantes de la Facultad de Odontología. UNMSM. 2014

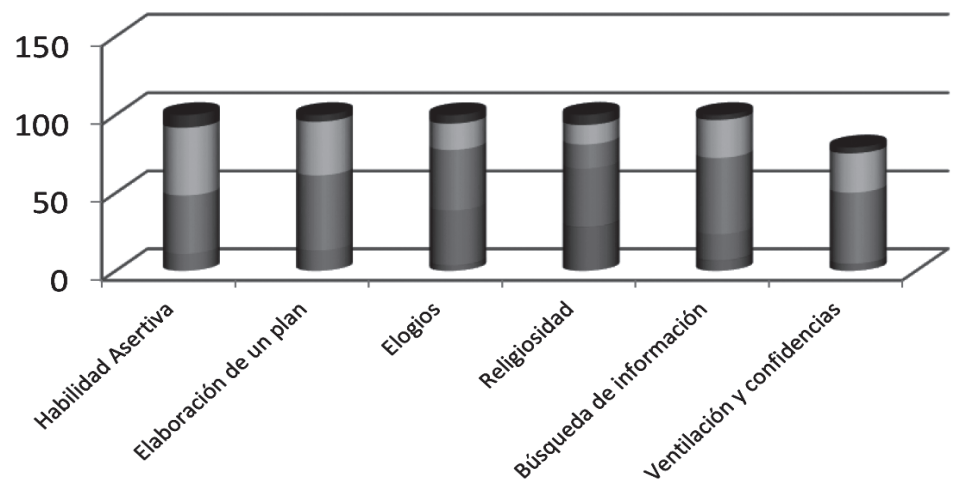

Siempre

Casi siempre

Algunas veces

Rara vez

nunca 
Tabla 4. Reacciones al estrés según año académico de los estudiantes de odontología de la UNMSM. 2014

\begin{tabular}{|c|c|c|c|c|c|c|}
\hline \multirow{2}{*}{ Reacciones al estrés } & & \multicolumn{5}{|c|}{ Año de studios } \\
\hline & & Primero & Segundo & Tercero & Cuarto & Quinto \\
\hline \multirow[t]{2}{*}{ Trastornos del sueño $\left(^{*}\right)$} & $\bar{x}$ & 2,16 & 2,66 & 2,93 & 2,28 & 2,5 \\
\hline & $\sigma$ & 1,068 & 1,111 & 0,998 & 0,974 & 1,062 \\
\hline \multirow[t]{2}{*}{ Fatiga crónica } & $\bar{x}$ & 3,14 & 2,9 & 3,2 & 3,17 & 3,33 \\
\hline & $\sigma$ & 0,976 & 1,081 & 1,091 & 0,971 & 1,047 \\
\hline \multirow[t]{2}{*}{ Dolores de cabeza o migraña } & $\bar{x}$ & 2,78 & 2,52 & 2,86 & 2,64 & 2,6 \\
\hline & $\sigma$ & 1,182 & 1,09 & 1,025 & 1,018 & 1,317 \\
\hline \multirow[t]{2}{*}{ Problemas digestivos, abdominales, diarrea } & $\bar{x}$ & 2,08 & 2,41 & 2,57 & 2,36 & 2,1 \\
\hline & $\sigma$ & 1,14 & 0,983 & 0,95 & 0,899 & 1,033 \\
\hline \multirow[t]{2}{*}{ Rascarse, morder uñas, frotarse } & $\bar{x}$ & 2,65 & 2,34 & 2,55 & 2,47 & 2,35 \\
\hline & $\sigma$ & 1,4 & 1,23 & 1,04 & 1,16 & 1,19 \\
\hline \multirow[t]{2}{*}{ Somnolencia, mayor necesidad de dormir } & $\bar{x}$ & 3,65 & 3,31 & 3,2 & 3,53 & 3,6 \\
\hline & $\sigma$ & 0,949 & 1,198 & 1,231 & 1 & 0,955 \\
\hline \multirow[t]{2}{*}{ Inquietud $\left({ }^{* *}\right)$} & $\bar{x}$ & 2,95 & 2,76 & 3,07 & 2,69 & 3,13 \\
\hline & $\sigma$ & 1,079 & 0,636 & 1,108 & 0,889 & 1,042 \\
\hline \multirow[t]{2}{*}{ Sentimientos de depresión y tristeza } & $\bar{x}$ & 2,62 & 2,55 & 2,61 & 2,64 & 3,48 \\
\hline & $\sigma$ & 1,063 & 0,827 & 0,97 & 1,046 & 4,909 \\
\hline \multirow[t]{2}{*}{ Ansiedad, angustia desesperación } & $\bar{x}$ & 2,84 & 2,86 & 2,66 & 2,78 & 2,68 \\
\hline & $\sigma$ & 1,068 & 1,026 & 1,219 & 0,989 & 1,118 \\
\hline \multirow[t]{2}{*}{ Problemas de concentración } & $\bar{x}$ & 3 & 2,86 & 2,89 & 2,81 & 2,98 \\
\hline & $\sigma$ & 1,027 & 0,833 & 0,689 & 1,009 & 1,025 \\
\hline \multirow[t]{2}{*}{ Sentimientos de agresividad o aumento de irritabilidad } & $\bar{x}$ & 2,68 & 2,38 & 2,75 & 2,36 & 2,4 \\
\hline & $\sigma$ & 1,18 & 0,862 & 0,892 & 0,899 & 1,033 \\
\hline \multirow[t]{2}{*}{ Conflicto o tendencia a polemizar o discutir $\left({ }^{* * *}\right)$} & $\bar{x}$ & 2,35 & 2,38 & 2,82 & 2,19 & 2,25 \\
\hline & $\sigma$ & 1,06 & 0,775 & 0,815 & 0,749 & 0,84 \\
\hline \multirow[t]{2}{*}{ Aislamiento } & $\bar{x}$ & 2,16 & 2,34 & 2,32 & 2,33 & 2,35 \\
\hline & $\sigma$ & 0,928 & 1,078 & 0,857 & 1,014 & 0,864 \\
\hline \multirow[t]{2}{*}{ Desgano } & $\bar{x}$ & 2,62 & 2,79 & 2,7 & 2,75 & 3,05 \\
\hline & $\sigma$ & 0,893 & 1,048 & 0,668 & 0,806 & 0,846 \\
\hline \multirow[t]{2}{*}{ Aumento o reducción de consumode alimentos $\left({ }^{* * * \star}\right)$} & $\bar{x}$ & 2,78 & 2,52 & 3,25 & 2,75 & 2,88 \\
\hline & $\sigma$ & 1,182 & 0,911 & 0,719 & 1,025 & 1,017 \\
\hline
\end{tabular}

$\left(^{*}\right)$ Significativo por ańo de estudio. $\left.K=26.38 \mathrm{p}=0.049 \quad{ }^{* *}\right)$ Significativo por ańo de estudio. $\mathrm{K}=32.38 \mathrm{p}=0.009$

$\left(^{* * *}\right)$ Significativo por año de estudio. $\left.K=30.84 \mathrm{p}=0.014{ }^{* * * *}\right)$ Significativo por año de estudio. $\mathrm{K}=29.53 \mathrm{p}=0.021$

Tabla 5. Rendimiento académico e intensidad del estrés según ańo de estudio de los estudiantes de odontología. 2014

\begin{tabular}{ccccccc}
\hline & \multicolumn{3}{c}{ Rendimiento académico* $^{*}$} & \multicolumn{4}{c}{ Intensidad del } & estrés \\
& $* *$ \\
Año & $\bar{X}$ & $\sigma$ & IC & $\bar{X}$ & $\sigma$ & IC \\
Primero & 12,96 & 1,41 & $12,46-13,46$ & 3,27 & 0,90 & $2,97-3,57$ \\
Segundo & 12,59 & 1,16 & $12,11-13,07$ & 3,31 & 1,00 & $2,93-3,69$ \\
Tercero & 13,29 & 0,78 & $13,05-13,24$ & 3,70 & 1,08 & $3,37-4,03$ \\
Cuarto & 13,55 & 0,84 & $13,24-13,87$ & 3.66 & 0.77 & $3,39-3,92$ \\
Quinto & 13,63 & 0,63 & $13,40-13,87$ & 3,35 & 0,80 & $3,09-3,61$ \\
\hline
\end{tabular}

${ }^{(*)} \mathrm{p}=0.000 \quad \mathrm{~F}=5,33 \quad\left({ }^{* *}\right) \mathrm{p}=0,100 \quad \mathrm{~K}=7,77$

Tabla 6. Correlación entre la intensidad del estrés y rendimiento académico

\begin{tabular}{lll}
\hline & $\begin{array}{l}\text { Coeficiente } \\
\text { Rho de Spearman }\end{array}$ & $\mathrm{P}$ \\
\hline $\begin{array}{l}\text { Nivel de estrés- } \\
\text { Rendimiento académico }\end{array}$ & 0.105 & 0.183 \\
\hline
\end{tabular}

Tabla 7. Modelo de regresión lineal para rendimiento académico

\begin{tabular}{lcccc}
\hline & $\begin{array}{c}\text { Coeficiente } \\
\text { Estandarizado } \\
\text { Beta }\end{array}$ & Significancia & \\
\hline $\begin{array}{l}\text { Habilidad } \\
\text { asertiva }\end{array}$ & 0.167 & 0.034 & & \\
\hline Model & $\mathrm{R}$ & $\mathrm{R}$ Square & $\begin{array}{c}\text { Model Summary } \\
\text { Adjusted R Square }\end{array}$ & $\begin{array}{c}\text { Std. Error of the } \\
\text { Estimate } \\
1\end{array}$ \\
\hline & $167^{\mathrm{a}}$ & .028 & .022 & 1.03244 \\
\hline
\end{tabular}


Estas manifestaciones son explicables por lo encontrado como principales estresores: sobrecarga de tarea y tiempo limitado que genera ansiedad, angustia y desesperación por sentir que no se cuenta con el tiempo suficiente para el cumplimiento de las tareas exigidas generándoles pocas horas de sueño y los consiguientes trastornos de sueńo y fatiga crónica.

El rendimiento académico de los estudiantes de odontología fue en promedio 13 , siendo bajo comparativamente, al promedio obtenido por estudiantes de otras facultades de odontología. Urge reflexionar sobre los alcances de este resultado. Podría ser que la sobrecarga de trabajos sumada a la repercusión física y psicológica que genera en el estudiante lo lleva a que no responde de mejor manera en su desempeńo académico, o ¡será que los procesos de evaluación no están midiendo adecuadamente el desempeńo y el logro académico del estudiante? Todos los estudiantes presentaron una intensidad de estrés en promedio entre 3 y 4 puntos, en todos los años de estudio, lo cual podría explicar la no asociación con el desempeño académico.

La habilidad asertiva, entendida como la capacidad para defender nuestras preferencias ideas o sentimientos sin dañar a otros, fue la principal estrategia de afrontamiento para abordar el estrés y la única que se relacionó directamente con el rendimiento académico de los estudiantes. Sin embargo este resultado difiere al de Velásquez ${ }^{10}$ quien encontró que la asertividad no está relacionada al rendimiento académico de estudiantes. Beltrán ${ }^{11}$ encontró frecuencia menores de esta habilidad como parte de estrategia de afrontamiento, difiriendo de lo encontrado en el presente estudio. Un punto importante en el hecho de manejar el estrés es poder defender lo que creemos correcto y/o demostrar que se ha desarrollado el proceso de aprendizaje, que se ha estudiado, cumplido con las actividades propuestas, entre otros. Esta habilidad es desarrollada por poco más del $50 \%$ de los estudiantes de odontología, siendo la estrategia que le permite afrontar las situaciones y reacciones de estrés a los cuáles están sometidos los estudiantes de odontología.

\section{Conclusiones}

Los principales estresores que se presentaron en los estudiantes de odontología fueron sobrecarga de tareas, el tiempo limitado y las evaluaciones, siendo la habilidad asertiva en el manejo del estrés el factor asociado y determinante en el rendimiento académico.

\section{Agradecimiento}

Al Vicerrectorado de Investigación por el financiamiento económico para el desarrollo del presente estudio.

\section{Referencias bibliográficas}

1. Berrío GN, Mazo ZR. Estrés Académico. Revista de Psicología. Universidad de Antioquia. 2011; 3(2): 65-82.

2. Díaz CS, Arrieta V. K, González M. F. Estrés académico y funcionalidad familiar en estudiantes de Odontología. Revista Cientifica Salud Uninorte. Barranquilla (Col.) 2014; 30 (2): 121-132

3. Casuso HJ. Estudio del estrés, engagement y rendimiento académico en estudiantes universitarios de ciencias de la salud. [Tesis Doctoral]. Facultad de Enfermería: Universidad de Málaga. Málaga. 2011.

4. Maruris RM y col. Factores estresantes asociados al hiperestrés en estudiantes universitarios de la ciudad de Chilpancingo, Guerrero, México. Summa Psicológica UST, 2012; 9(1):15-21.

5. Bedoya SA, Perea M, Ormeño R. Evaluación de niveles, situaciones generadoras y manifestaciones de estrés académico en alumnos de tercer y cuarto año de una Facultad de Estomatología. Rev Estomatol Herediana 2006; 16(1): 15 - 20.

6. Vallejo-Agudelo E, Martínez-Sánchez L, Agudelo-Vélez C. Estrés: determinante genérico del desempeño académico. Revista de educación y desarrollo. México. 2013; 26(Julio-septiembre):89-93

7. García-R R, Pérez-González F, Pérez-Blasco J, Natividad L. Evaluación del estrés académico en estudiantes de nueva incorporación a la universidad. Revista Latinoamericana de Psicología. Bogotá. Colombia. 2012; 44 (2): 143-154.

8. Del Toro AA, Gorguet PM, Pérez IY, Ramos GD. Estrés académico en estudiantes de medicina de primer año con bajo rendimiento escolar. Medisan. 2011;15(1):17.

9. Alves E. y Acevedo R. La evaluación cualitativa. Reflexión para transformación de la realidad educativa. 2a ed. Caracas: Petroglifo Producciones. 2002: 156p.

10. Velásquez CC y col. Bienestar psicológico, asertividad y rendimiento académico en estudiantes universitarios Sanmarquinos. Revista IIPSI Facultad de psicología. UNMSM. 2008. 11(2):139-152.

11. Beltrán HK. Estrada CM. Martínez BA, Navarro AT. Como afecta y se relaciona el estrés con el rendimiento académico de estudiantes de segundo año de la carrera de Medicina del CUM USAC, durante el mes de mayo 2010. Universidad San Carlos de Guatemala. Unidad Académica de Salud Pública. [Internet] 2014 [citado 25 junio 2014] Disponible en: http://digi.usac.edu.gt/encuentro2011/uploads/encuentro2010/ insumos/Estres\%20en \%20estudiantes $\% 20$ del\%20CUM\%20 INFORME\%20FINAL.docx. 